\title{
Playing along and playing for on Twitch: Livestreaming from tandem play to performance
}

\author{
Rainforest Scully-Blaker, Jason Begy, Mia Consalvo \& Sarah Christina Ganzon \\ Concordia University \\ Department of Communication Studies \\ Montreal, Canada \\ fscullyblaker@gmail.com \& mia.consalvo@concordia.ca
}

\begin{abstract}
This paper is an analysis of individuals who livestream gameplay on Twitch. Two core concepts 'playing along' and 'playing for' - are put forth as two poles to a continuum to better discuss tandem play in the context of livestreaming. From an analysis of participants' exit interviews and observations of larger Twitch streams, it is shown that livestreaming is a form of tandem play, but only to a point. As audiences grow, 'playing along' becomes difficult for streamers. The 'ceiling' of tandem play is reached when a streamer is so focused on entertaining the largest number of people possible that they are no longer playing along with their spectators, but only playing for them.
\end{abstract}

\section{Introduction}

From early game machines like Tennis for Two [10] up to contemporary MMOGs, playing together has always been a part of videogame history and indeed the history of play itself. It is not uncommon to see games that are dubbed 'single player' experiences being consumed by multiple individuals sharing a couch or other common 'space' such as the virtual performance created by livestreaming one's play. However, game studies as a field has yet to investigate this phenomenon in depth. It was because of this that a preliminary investigation of what has now been dubbed tandem play, or "when two or more players engage with a single-player game together, moving through the game with a variety of potential motives" was undertaken [1].

In that paper we argue that, "tandem play is not an activity we invented, even if we are coining this term to describe it," we are simply giving a name to "a specific style of play with a long history and contemporary expression" with a view to examining the relation between performativity and different play contexts [1]. As such, for this study, fifteen individuals were split into two groups to play
Bioware's RPG Dragon Age: Inquisition [5] - five pairs that played Dragon Age on Xbox One, each pair sharing the same character and passing the controller back and forth; and five individuals that played the game on PC, livestreaming their play on Twitch.tv. Whereas elsewhere we make the case that our couch co-op sessions demonstrate the many ways that tandem play includes "collaboration [that] affects game play," it is the livestreamers that will be our primary focus in this paper [2]. Taking the stance that livestreaming represents another form of tandem play, this paper asks how tandem play is affected by the various limitations and affordances of the live streaming of gameplay via Twitch.

Twitch.tv is a free platform and website that attracts millions of individuals who broadcast their own personal 'channel' to the world, showing live video of themselves as they play and talk, as well as a continuous stream of their gameplay that anyone can view. Live streaming of both multiplayer and single player games has become a key way that individuals and groups now experience the act of playing games. Sites like Twitch.tv offer individuals simple ways to broadcast live video of their gameplay along with audio and/or video images of themselves as they play. Viewers, who number from the single digits to the thousands or millions, can also chat with streamers and each other, or simply enjoy the commentary that streamers provide. Videogame live streaming has become lucrative: Twitch.tv launched in 2011 and Amazon bought the service in 2014 for $\$ 970$ million [1].

\section{Review of literature}

There is much literature built around the discussion of play and spectatorship, but relatively little that examines videogame livestreaming. Mehdi Kaytoue et al authored one of the earliest efforts to study livestreaming via an in-depth analysis of data that 
was collected over "more than 100 days" pertaining to how particular games and streamers grow in popularity [11]. The piece offers insight into how great an impact major gaming tournaments have had on the growth of both Twitch as a community ${ }^{1}$ made up of "actors" ("the spectators, the pro-gamers, their sponsors, the game publishers, etc.") as well as individual streamers' channels as micro-communities that overlap and interact with one another. Yet Kaytoue et al's piece leaves untouched many questions about the figure of the livestreamer and the practice of livestreaming that this study picks up, including how smaller streamers view their relationship with (any) viewers they might attract.

William A. Hamilton et al are also concerned with the growth of Twitch, but with a focus on how communities form around certain streams [10]. They undertake an ethnographic investigation of "the emergence of communities amidst live streaming on Twitch." They characterize Twitch as 'third places,' or "locations for people to come together, form, and maintain communities" that are distinct from home or work spaces. They also situate Twitch communities within D. W. McMillan and D. M. Chavis' concept of the "sense of community" which is made up of four aspects: "membership, influence, fulfillment of needs, and emotional connection." These concepts were helpful for thinking through our own observations of the livestreaming practice, yet pushed us to ask how streamers themselves interacted with these communities.

To move us closer to such questions, we turned to work on play and spectatorship via the more established platform of YouTube, where gamers have had more time to establish practices and conventions for what to post and how to interact with individuals who might 'subscribe' to their YouTube channel. In his article, "The socio-technical architecture of digital labor: Converting play into YouTube money," Hector Postigo conducts a study of "20 commentators and their communities" [15]. Although he begins by watching videos and reading comments, he ultimately transitions into starting "[his] own channel, and [making] commentary videos of [his] own."

Through this approach, Postigo was able to examine YouTube as an infrastructure of "affordances" that "create a set of probable uses/meanings for YouTube, most of which are undertaken as social practice" while simultaneously

\footnotetext{
${ }^{1}$ It should be noted that to refer to Twitch solely as a community in 2015 is much more of an oversimplification than it was in 2012.
}

serving the site's "business interests." Commentators need to stake great sums of money on their success through purchasing the hardware and software necessary to be a content creator. As such, there is pressure to convert this investment into footage that can be deemed "YouTube-worthy," that is, gameplay that "involves not only talent, but also the use of a number of technological and social structures that convey competitive advantage." As we will show when discussing Twitch and our subjects' exit interviews, a similar pressure seems to be exerted on the livestreamer to produce content that is 'Twitchworthy,' but what this entails and how it can be accomplished when game footage is not carefully sculpted and edited in advance of its broadcast will be discussed.

Daniel Smith's piece, "Charlie is so 'English'like: nationality and the branded celebrity person in the age of YouTube," plays with the video hosting site's well-known invitation to 'Broadcast Yourself' to discuss how YouTube personality, Charlie McDonnell's "self-commodification is accomplished through the development of a persona" [15]. Smith focuses particularly on one YouTube personality's expression of an "Englishness" that evokes "mythic elements of national identity" to carve out a following for himself on the video hosting site. And while notions of national identity were not mentioned by our subjects during their streaming, Smith's piece remains relevant for its discussion of persona and performativity. Indeed, our subjects had total control over what to show their viewers and how to interact with the game they streamed, and yet subjects often admitted to feeling as though they were performing for an audience rather than playing for themselves.

That idea of playing for an audience online via a live setting has also been studied in a different yet complementary context by Theresa Senft. In Camgirls [14], Senft offers an "ethnographic and critical study of one generation of camgirls and their viewers from 2000 to 2004" by engaging in interviews with performers, conducting participant observation of webcam feeds, and taking part in the camgirl practice itself. Beyond the work's obvious emphasis on performativity and spectatorship, Senft's work was also tied to our own through the author's concern with "the folly of broadcasting [her] image to anyone with a computer and an interest in watching," something that was felt by several of our participants. Beyond this text's relevance as a discussion of spectatorship and performativity in the online sphere, it was also interesting due to the fact that it has become increasingly common to find 
camgirls turning to Twitch and livestreaming gameplay as another means of revenue. While this phenomenon and the various responses it has received from inside and outside of Twitch communities merits papers unto itself, comparing Senft's findings to our own deepened our understanding of practices that involve sharing oneself with an online audience by offering a treatment of online performativity in a non-gaming context.

Finally, to broaden our understandings of the potential these practices reveal beyond gameplay, we drew from Ducheneaut et al's study of social TV, and how an audience can develop conventions and expectations for interaction while viewing that provides for a richer experience overall [6]. Indeed they argue such television viewing can result in "a kind of "vicarious audience play"' which perhaps holds true for viewers of Twitch streams as well. While we did not interview viewers of our streams, our study can stand as an early step toward better understanding how Twitch streamers can facilitate such processes, as well as when/if they might not.

\section{Methods}

To gather participants, we advertised a play study based out of our lab that involved the then-new game, Dragon Age: Inquisition, in which players assume the role of the Inquisitor, forging alliances and making sacrifices to save the world from a cataclysm. The game was selected for two main reasons. As mentioned above, at the time of the study, the game was still relatively new, increasing the likelihood that our livestream would draw viewers that were interested in learning about the title. As well, the Dragon Age series is known for putting players into many situations in which they must make decisions, be they logistical choices like which quest to do next or major ethical decisions such as siding with one faction over another. It was believed that such choices might invite our participants to interact with others, as they would have to make multiple decisions during their gameplay sessions.

Subjects were solicited through posters placed around campus as well as through emails sent to two local game studies listservs. Those of us who were teaching classes that semester also announced the call for participants to their students and one member of the research team also recruited a subject through word of mouth. In total, we had fifteen participants, seven of which identified as female and eight which identified as male. Ages ranged from 20 to 42 . Once recruited, participants were asked whether they would prefer the couch co-op or livestreaming segment of the study and 5 of those that requested to stream their gameplay (or at least stated that they had no preference either way) were scheduled for three ninety-minute play sessions. Our livestreaming group again ranged from 20 to 42 years old and consisted of 3 women and 2 men. As we learned later, only one of five subjects had ever livestreamed before while another had experience with doing Let's Plays and uploading their gameplay on YouTube.

Participants were asked to personalize their stream layout, most notably by deciding whether they wanted to broadcast either microphone audio or a webcam feed, or both. While there was some initial reluctance on the part of some subjects to include a 'facecam,' all subjects did agree to use the microphone and, by their third and final sessions, all but one of the subjects were broadcasting a webcam feed in addition to their gameplay. The Twitch chat for the stream was left open in an adjacent monitor for the player to read and, if they so desired, reply to in whatever manner they saw fit. For most subjects this consisted of replying through the microphone, although two subjects occasionally interrupted their gameplay to type their replies into the chat window itself.

Each gameplay session was physically attended by at least two of the researchers involved in this project. We would generally seat ourselves at nearby computers with the stream open in front of us in order to detect technical problems or offer our players guidance, either orally or, on occasion, through the Twitch chat. We did not view these interactions as problematic, since the goal of this study was not to garner as many Twitch viewers as possible, but rather to simulate a normal streaming experience and study participant behaviour. Taking a cue from Fine's "participant-as-researcher," then, on days when we had no external audience, we saw no issue with becoming involved in the Twitch chat ourselves [7].

During the gameplay sessions, we would also take notes of the player's activities both-in game and in the physical space of the lab. Upon finishing their third and final play sessions, participants would take part in a semistandardized exit interview. Questions ranged from a general overview of the play sessions, whether they liked their created character or the game itself, to pointed questions about the streaming experience and how it may have altered their play style or their personal behaviour. 
Couch co-op sessions were conducted similarly with the major difference of course being that rather than having an audience made up of Twitch viewers, these participants were only observed by each other and attending researchers. Participants played the game on console, passing the controller back and forth with whatever degree of regularity they mutually decided upon. Pairs were generally decided upon based on availability, but one pair, a couple, knew each other before the study began.

Once this phase of the study was concluded, the interviews themselves were transcribed and our participants' answers were revisited with a view to picking out notable trends that related to our working understanding of tandem play. Along with the aforementioned literature on livestreaming, performativity, spectatorship, and play, the observations that emerged from revisiting the interview transcripts, combined with additional observation of Twitch.tv, its limitations, affordances, and trends are what form the basis of our findings in this paper. ${ }^{2}$

\section{"Twitch-worthy" gameplay}

When Hector Postigo studied some of YouTube's gaming content creators, he found that these individuals took it upon themselves to produce what he calls "YouTube worthy" footage, which, "at its most extreme" is more akin to "a well-orchestrated performance" than actual gameplay [13]. While some people who upload highlight videos to YouTube are under contract with Machinima or similar companies, those that are not still put effort into creating, collating, and refining hours of recorded gameplay into an entertaining final product: a video that generally takes much more time to produce than it does to watch. And while this desire for entertainment value has carried over to Twitch streamers, a tension emerges in livestreaming that is not felt in previous modes of presenting gameplay footage. For indeed, how is one to produce what we might call 'Twitch worthy' content given the inherent lack of any ability to edit their gameplay before it is consumed by an audience?

As far as good gameplay is concerned, what is valued on a livestreaming site like Twitch is different from what is valued on a video hosting site like YouTube, to an extent. YouTube videos are often a

\footnotetext{
${ }^{2}$ Due to the experimental nature of the project at this early stage, later observations were anecdotal, hence their exclusion from any broader mention in the methods section.
}

'greatest hits' of particular play sessions - the highlight reel the morning after a sporting event while Twitch offers viewers a window into the 'live and uncut' play sessions in their entirety - the sporting event itself, with all the high and low moments. To watch a YouTube video, or even an archived Twitch video of a DoTA 2 match is to watch a fait accompli - as eSports grows in popularity, it becomes increasingly difficult to attempt to watch the recording of a tournament after the fact 'spoiler-free.' Watching competitive gaming live, however, allows the viewer to take in the full experience of the ludic athleticism that unfolds, although there is much more to watch and much more downtime between matches. As far as eSports goes, each mode of watching professional gameplay has its place, but what of noncompetitive gameplay?

As Postigo notes, some YouTube commentators "refer to themselves as 'directors,' a nod to their shifting identity as they move from being "just a gamer' $[\ldots]$ to a professional who is creating a genre of entertainment" [13]. Even YouTube commentators that play single-player games or whose skills are not on par with the world of eSports are still able to garner millions of views on a given video because they cater to certain genre conventions that make their content as entertaining as possible for the greatest number of viewers by 'playing for' the masses. For instance, some of the most popular YouTube gaming commentators (Game Grumps, PewDiePie, et al) rely on comedic timing and memetic inside jokes to develop a fanbase while others play team-based games with friends and stage things such that they can take a starring role in the production. Postigo also cites the example of the console "giveaway," where content creators spend thousands of dollars on gaming hardware to reward random subscribers with the hope being that their loyal viewership will ultimately yield a profit. And while livestreamers can and often do give humourous commentary to their gameplay or play with their friends, the nature of Twitch-worthy content is fundamentally different from the gameplay seen on YouTube. Short of cutting off one's Twitch feed altogether, the livestreamer cannot interrupt or otherwise cut together gameplay into a set of highlights for potentially thousands of viewers. ${ }^{3}$ Instead, the livestreamer has a problem that the YouTube personality does not - how to minimize the lulls in gameplay and fill whatever inevitable gaps in

\footnotetext{
${ }^{3}$ After playing live there is the option to post a day's segment and 'highlight' it afterward, and many Twitch streamers also have a YouTube channel where these highlights are also uploaded. Such elements were outside the scope of this study.
} 
the action that might occur.

Despite having no more than a handful of viewers at a time, it was clear that our streamers felt this same pressure to 'play for' an audience during their play sessions. Alice (all names used here are pseudonyms), the subject who had made several Let's Plays and uploaded them to YouTube, informed us at the beginning of her first play session that although she would use the webcam and microphone, this did not mean that we should expect her to offer commentary over her gameplay since she tended to be a quiet gamer. Within minutes of sitting down and going live, however, she quickly became involved in some of the most extensive exchanges with people in the Twitch chat. When we asked her about this after the final play session, she explained:

\section{I felt more outgoing all of the sudden. [Livestreaming]'s something I've been thinking of doing. On my YouTube channel, you never see my face, you only hear me talking. [...] Once I found myself more comfortable with all the stuff I thought what the hell, it's fun to talk to people.}

The early stages of Dragon Age: Inquisition are fairly straightforward, with a lot of wandering the picturesque countryside and completing simple quests. Alice had played the game already, and was therefore able to anticipate the lulls in gameplay and fill them with commentary. Not only did this make her play sessions more interactive and entertaining for her viewers, but, as Alice asserted, it also made her second playthrough of Inquisition more enjoyable.

Although we initially thought that it was Alice's Dragon Age expertise that allowed for livestreaming to make what was otherwise a familiar play experience more interesting, we soon found that her reaction to having an audience was not unique. Another of our participants, Rick, also felt the imperative to be entertaining:

\section{So [one of] the things that I noticed is that, I tend to talk a little bit more. Like, when I'm home by myself I don't talk. You feel like you have an audience. So you try to interact with them.}

Unlike Alice, who had prior knowledge of the game's pacing and enough viewers ${ }^{4}$ that she could largely space her commentary around what was happening in the Twitch chat, Rick found himself speaking even if there had been no activity in chat. And although he was experiencing Inquisition for the first time, Rick still found himself striving to show viewers more interesting aspects of the gameplay, 'playing for' those that were interested in watching. When asked if he would have played differently at home, he replied:

I think I might put more time in
reading stuff. Not the long [texts],
because there are some that are
really long. But I think I might slow
down a bit more and figure out how
everything is going.

Because he was streaming, as Rick put it, "I would show people what I would want to watch." From this it is clear that, although it is not possible to apply the same highlight-based approach to livestreamed content as it is to YouTube videos, there is still an editorial practice at work on Twitch.

Playing for the crowd was not the only thing on our livestreamers' minds, however. Tandem play is a combination of playing for people as well as playing along with them. Even with a very small audience, participants felt as though they were playing for their audience, both in the sense that they had an imperative to be entertaining and in the sense that they felt the need to make choices that were more interesting. Yet how were they playing with their viewers? Livestreamed gameplay demands a certain entertainment value which affected not only our subjects' choosing to provide audio commentary or seek out interesting gameplay, but also impacted the decisions that they made while playing the game.

\section{Crowd-sourced choices: Playing for an audience}

In parallel with the five sets of livestreaming sessions that we conducted with Dragon Age: Inquisition, ten other participants were paired off and instructed to play the game in a 'couch co-op' style [2]. Our exit interviews found that, unsurprisingly, all of these participants made their decisions with their

\footnotetext{
${ }^{4}$ The number of viewers ranged between 0-10 for most broadcasts, however we noted that Alice had a particularly robust chat for one of her play sessions. It is not clear if anything specific prompted this larger audience since streams were always broadcast at approximately the same time of day and we always advertised them through the lab's Twitter account.
} 
partner (and often the researcher-spectators) in mind. For instance, one participant, Oscar, said of the character creation process with his partner:

\begin{abstract}
[Allen] had played through the game as a mage already, so we wanted - or he at least wanted to experience something new which again I was alright with and so we chose a warrior and he had no real qualms on the race except he didn't want to play an elf, which was fine.
\end{abstract}

All couch co-op participants admitted to altering their playstyle or making decisions for the sake of entertaining or at least collaborating with their partner. And the same can largely be said about the livestreaming participants with regards to their audience.

One of the major functions of the Twitch chat for participants, particularly those that had never touched a Dragon Age game before, was a forum to ask questions about how to access menus or where to find particular objectives. Over time, however, both groups--those that had played through Inquisition already and those who had not-- gravitated towards the possibility of there being an audience to impact gameplay decisions. As one participant recalled when asked whether the chat had affected his play:

\begin{abstract}
Because I was being streamed, I think that maybe I was a little bit more inclined to be like, 'what's some cool side things I can do right now'. I think I might've subconsciously figured, people who are watching me play this game probably might already know what the main questline in this game looks like. They probably won't be very interested if they see me going through the story because they already know what's going to happen. So maybe let's go see something funky and alternative that I can do in this game that will keep them entertained.
\end{abstract}

Despite not receiving any prompting from the chat to suggest that certain things were more or less interesting to watch, this participant imagined an audience and strove to play in such a way as to avoid boring them. This is supported by data showing other times when our streamers were playing for the audience, playing along with real as well as hypothetical viewers.

Another participant who did receive explicit suggestions from the chat recalled that, when they were at a loss for where to explore next, "someone said 'I like the Storm Coast, even if it can feel dreary.' So I said let's go check that." Regardless of whether it was the decision to undertake particular side quests, or the decision to travel to a new area, subjects directed their play around what the chat might implicitly or explicitly want them to do.

Although the interchange between our streamers and their chat was not as seamless as it was between our co-op pairs (more than one participant noted the delay that existed between their actions and the chat's response - one of the more well-known issues with Twitch and other livestreaming sites), we can see that livestreaming encourages even first-time streamers to interact with viewers in a manner similar to the way that playing a game with others in the room encourages one to interact with their physical audience. On more than one occasion, our participants likened the livestreaming practice to times they had done couch co-op-like activities in the past. Whether it was playing with siblings as was the case for one participant:

I would tell [my brother], 'Oh, I'm playing such and such' and he would stick his head in the room and comment about certain things or he world actively say oh I want to see you play the opening sequence and see what you have to say and stuff like that. I'll do live texting of something to my brother because he knows what's happening he'll have the dramatic irony, I'll ask him a question and he'll just laugh and respond.

or whether it was playing with friends or in a more public setting like an arcade, many of our livestreamers were familiar with playing while being watched. One participant noted, "I am used to having people around me, looking over my shoulder while I played - friends. Growing up especially in games rooms I always had people around me. So [livesteaming was] new in a way, but not really."

It became clear to us that, more than being a similar practice to couch co-op, these livestreaming sessions represented another form of tandem play. In 
fact, a large part of what appealed to our subjects about livestreaming was those elements that cemented it as a tandem play practice. Participants repeatedly felt that they were "playing with other people," and that these people in chat were afforded the opportunity to "influence in real-time what [was] happening" in collaboration with the player. All participants agreed that this made the experience more enjoyable for them. But beyond asserting that livestreaming is akin to two or more players engaging with a single-player game together, how does understanding Twitch as a platform for tandem play allow us to deepen our understanding of the place of livestreaming in game culture? To answer this, let us now shift the tandem play lens from the experiences of our participants to larger, more established Twitch communities.

\section{The ceiling of tandem play on Twitch}

One of the questions we asked our participants was why they thought that people would livestream their gameplay. With eSports growing in popularity and earning more money for game companies and eathletes alike and with some of YouTube's gaming personalities making yearly revenues in the millions, it is unsurprising that one of our participants began by answering:

The cynical thing that comes to
mind is that a lot of people feel like
they can make a profit off of
streaming. Because a lot of people
have really profited off of having
sort of really eccentric, likeable
personalities when it comes to Let's
Play videos or streaming. Any of
these opportunities to express
yourself on the Internet that these
video sources have provided us
with. A lot of people think 'I can get
payments off the Internet doing this
and I don't have to do a "real
job"...

This point of view is by no means baseless. In outlining the architecture and affordances of YouTube, Postigo makes certain to highlight the importance of "favoriting a video," "the subscription system," and "the advertising system" as means of converting play into a self-sustaining form of digital labour and it should come as no surprise that Twitch has similar mechanisms in place [13].
Twitch actually takes the standard subscription system one step further by charging viewers five dollars to subscribe to a favourite streamer. But unlike YouTube, where a subscription's primary function is to receive notifications whenever a new video is published, Twitch subscriptions come with certain added privileges. ${ }^{5}$ These privileges vary from channel to channel, but include access to unique emoticons, the opportunity to play with or against a Twitch personality (in what are called 'sub-games'), and, perhaps most notably for our purposes, access to the subscriber-only chat. As Hamilton, Garretson, and Kerne point out, the major draw of the 'sub-only' chat is that it serves to "cut down on the inherent noise of having thousands of people together in a single chat room" [9]. The fact that the privilege of participating in chat at all times is something for which people are willing to pay and that Twitch personalities can make some profit off of this does not go unnoticed by would-be streamers. Because Twitch and YouTube's very structures afford ready access to profit for what might appear to some to consist only of playing videogames, the 'cynical' answer given by the aforementioned participant is likely a major reason that some people who have not streamed before may become interested in the practice.

And yet, after having streamed their gameplay for only 4.5 hours, our participants tended to offer socially based reasons for why people might want to stream their gameplay, or why they might want to continue streaming theirs. ${ }^{6}$ Even the participant who initially cited profit as a major reason for streaming was quick to add:

But I also think that there are some
people who, I dunno, they kind of
get a kick off of expressing
themselves. They feel like maybe
they sort of want to show everyone
else what it is they're doing and
they only get a few hours a day to
game because they have something
else that they have to do in life, so
might as well combine both these
things.

\footnotetext{
${ }^{5}$ Twitch also has a free 'follow' button that, similar to subscribing to YouTube channels, makes it easier to track when a particular streamer goes live.

${ }^{6}$ This study was the first exposure to Twitch that some of our participants had. Many cited watching Twitch and Dragon Age: Inquisition videos or livestreams in preparation for their play sessions. Future research may benefit from studying more seasoned Twitch streamers.
} 
It did not take long for our participants to realize that there can be something more to livestreaming than profit. From our work thus far, we would argue that this 'something more' is the tandem play experience and to justify this claim, we will conclude by expanding our scope from our five participants and relate tandem play to larger Twitch communities and practices to investigate whether tandem play occurs there as well.

Despite the fact that the viewer count for our livestreaming sessions was generally less than ten, our participants felt that they were both 'playing for' and 'playing along' with their viewers. The fact that they were broadcasting their gameplay of Dragon Age: Inquisition for an actual or hypothetical 'someone' altered not only the way they carried themselves, but also the way that they played the game itself. However, it can be objected that it was precisely because the view count was so low that our participants felt engaged. After all, it is only with more viewers that we see faster-moving chats that tend towards becoming the 'noise' that Hamilton et al cite as a major appeal to subscriber-only mode. Perhaps having fewer messages to read allowed for a more intimate streaming experience. Although securing a large audience was something we simply could not do in our experiment, this must still be a part of our analysis.

In their 2015 piece, "Live-Streaming Changes the (Video) Game," Smith, Obrist, and Wright classify livestreamers into several major 'communities:' $e$ sports, or those who play games "competitively," speedrunners, or those who attempt to "complete a game as fast as possible," and Let's Players, whose play focuses more on being "entertaining" than on competition or a particular set of skills [16]. While all of these communities are made up of channels that have audiences of different sizes, it can generally be seen, both from our anecdotal observations of Twitch and from the research conducted by Kaytoue et al that eSports channels generally have the largest audiences. This is followed by a handful of prominent Let's Players and speedrunners with less high-profile members of the speedrunning community generally outranking lesser-known Let's Players by a slight margin, likely due to the greater novelty of their play. We have already discussed how Let's Players may impose the sub-only chat on their streams to ensure that their connection with a dedicated segment of the audience can occur, and it is clear that eSports cannot be an instance of tandem play since all competitive games in this genre are multiplayer experiences. As such, this paper will focus on the speedrunning community in discussing larger audiences on Twitch.

As Smith et al assert, a large part of speedrunning as a practice "encompasses exploiting bugs in a game to skip as much game content as possible" [16]. As such, there exist pages of forum posts and hours of YouTube videos discussing glitches and showcasing different approaches to pulling off tricks to get the time it takes to complete a game as quickly as possible. With the growth of Twitch, this community effort to beat games faster has moved into livestreaming as well. Indeed, tuning in to a speedrunning stream is not only an opportunity to take in technical and entertaining play, but it also offers would-be runners a forum to ask the streamer questions or even engage in theorycrafting about why a particular glitch works or how the supposedly optimal route through a game might be improved [4].

As a result, the level of streamer-viewer interaction on speedrunning channels is generally quite high. Veterans and fledgling speedrunners alike engage in a dialogue with the streamer and "play along' with streamers much more explicitly than other livestreaming audiences, be it by suggesting changes to how a game is run, through prompting the runner to demonstrate how to practice particular exploits, or even offering the streamer tips on how to improve. We would argue that since, as Smith et al observe, "finding and exploiting bugs is a community effort," speedrunning livestreams are often fertile ground for the observation of tandem play, even when viewer counts climb into the thousands. However, there still does seem to be a ceiling on the amount of people that can be a part of a tandem play experience on Twitch [16]. ${ }^{7}$ Once a speedrunning stream reaches a certain level of viewership, the runner's focus may shift more into playing for his or her audience as a whole rather than answering individual questions or testing individual theories. As a streamer shifts further towards the 'playing for' pole of the tandem play spectrum, we would argue that a ceiling is reached and tandem play no longer occurs. While our thoughts on this ceiling are only informed suspicions based on our own experiences watching Twitch streams, we feel that, these suspicions stand as fruitful directions that future

\footnotetext{
${ }^{7}$ The simultaneity afforded by livestreaming seems mandatory for tandem play to occur in an online setting. While there may be exceptions, we cannot help but feel that there is a difference between a speedrunning Twitch chat and a speedrunning forum as far as tandem play is concerned. Discovering glitches live rather than via correspondence is what cements speedrunning livestreams as unique places to look for tandem play in action.
} 
scholarship on tandem play and livestreaming can take.

Twitch as a platform offers the potential for profit to prominent content creators by offering viewers the chance to become prominent in their own right. One feeds into the other because as a Twitch streamer becomes more popular and more people tune in, the incentive to pay for subscriber status is a better investment. By purchasing the subscriber status, one is guaranteed, at the very least, that one's 'voice is heard' above non-paying members of chat. Even if sub-only mode is not enabled, subscribers have special icons next to their usernames in the chat window as well as access to exclusive emoticons, which makes distinguishing them from nonsubscribers easy to do.

Streamers do not immediately have access to collecting subscribers or having tailor-made emoticons added to the Twitch chat lexicon, however. These privileges are earned through demonstrating that one's gameplay consistently draws a certain number of concurrent viewers. For people that wish to livestream for a living, this means that they must go the route of Postigo's YouTube content creators and play for an audience, incentivizing viewers to tune in through a combination of an entertaining stream persona and high-level gameplay as well as through giveaways or 'sub games.'

As a result, it seems, at some point in the cycle of gaining viewers by being entertaining and receptive to one's spectators (i.e. through practicing a form of tandem play), streamers possibly transition to an increasingly impersonal approach to their channel as the chat becomes larger and more unreadable. At this point, only the subscribers can claim to have any particular sense of 'playing along' with streamers, but whether or not the streamer cultivates this sort of interaction in their broadcasts likely varies. The 'ceiling' of tandem play is reached when a streamer is so focused on entertaining the largest number of people possible that they are no longer playing along with their spectators, but only playing for them. It is unclear as to whether there is an exact number of viewers at which this might tend to occur, but the spectrum of 'playing along' and 'playing for' on Twitch - the threshold between tandem play and pure performance - should be explored further. By framing livestreaming in this way and categorizing channels based on what extent they encourage tandem play to occur, major Twitch communities of practice like those described by Smith et al are made distinct from one another and, even within these larger communities, a gradient exists:

Let's Players on Twitch at all levels of popularity are most concerned with tandem play in the way that it engages their audience. Though it seems the initial appeal of streaming in this way is the sense of playing along with a few spectators, as a Let's Play channel grows in popularity, the tendency seems to be that streamers engage less with individuals and more with their mass of followers, with some attention paid to subscribers, until tandem play is no longer a focus.

Speedrunners on Twitch tend to start streaming with a view to playing along with their viewers as well, but there is also a broader sense of playing along with the entire speedrunning community. These streamers are most concerned with tandem play as a mode of theorycrafting, teaching, and learning. Streamers and spectators actively collaborate on discovering new tricks and helping new runners get better [4]. As is the case with Twitch Let's Players, a larger audience might mean that there is less opportunity for this tandem play to occur, but speedrunners are more of a tightly-knit community than Let's Players ${ }^{8}$ which lowers the likelihood that streamers shift from playing along with an audience to playing for them. ${ }^{9}$

\section{Conclusion: Playing for the masses}

This paper began by asking how tandem play as a practice was affected by the affordances and constraints of a platform such as Twitch. By first conceptualizing these livestreaming practices as forms of tandem play, we offer a means to distinguish between the various communities of practice that exist on Twitch while simultaneously offering a way to analyze individual channels as micro-communities at a stage in game scholarship when understanding livestreaming is more important than ever before. Prior research has focused mainly on successful streamers and large stream communities, which are the elements of Twitch that

\footnotetext{
${ }^{8}$ Postigo does note that on YouTube, Let's Players do create communities in a sense by following one another and sharing fans, these strike us more as business partnerships than organic gaming communities.

${ }^{9}$ A notable exception would be major speedrunning events like Awesome Games Done Quick, where many participants gather and complete games for charity. Like most eSports events, the actual players do not have access to the Twitch chat while they perform, instead offering a generically entertaining commentary for all who are watching. But even here, there are elements of 'playing along' that can be found.
} 
garner the most popular attention, but which only form part of the streaming picture. Many streamers only see viewer counts in the double or triple digits, or often even fewer. In our explorations of Twitch, it was not uncommon to find channels with viewer counts of 1 or even zero. While not attracting media attention or financial gains, such streamers also form part of livestreaming culture and deserve attention and study. Our research shows that such streamers also see their role as being entertaining and playing with others, even if no one may be immediately present to witness that activity.

Our research also speaks to newer channels that Twitch offers under the "Creative" theme, which feature individuals engaging in multiple activities such as making digital and analog art, playing guitar and cooking. This new form of 'social viewing' is a hybrid of entertainment and participant interaction with the host as well as amongst the community that forms. Better understanding of how gameplay streamers conceptualize and enact their role with their viewers - and how that role changes based on the size of one's viewership - can help us understand how these "creative" streamers are also functioning. And as Twitch continues to remain profitable and competitors are launched, understanding how streamers engage with their viewers will become vital across not just entertainment but likely for educational, political and scientific contexts as well.

Twitch only continues to cement itself at the centre of contemporary game culture. More and more major events are streamed on the site and the emergent gameplay practices that are showcased there are brought to the attention of the game industry itself as e-athletes continue to innovate in their interactions with games and develop competitive metagames, livestreamed gameplay informs game design. The Twitch interface has even become a game in itself on some streams, such as Twitch Plays Pokémon perhaps the most extreme example of tandem play on Twitch to date - where, at one time, hundreds of thousands of viewers successfully completed Pokémon: Red Version by entering millions of button inputs in the chat window.

Phenomena such as this, the nature of Twitch as a platform that facilitates the occurrence of tandem play, and how this, in turn, makes recognizable the numerous communities of practice that exist on the site (many of which were not discussed here) are all avenues of scholarly investigation that should be further explored if we seek a better understanding of livestreaming's place in game culture.

\section{References}

[1] [Redacted 1]

[2] [Redacted 2]

[3] [Redacted 3]

[4] [Redacted 4]

[5] Bioware. (2014.) Dragon Age: Inquisition. Electronic Arts. Xbox One.

[6] Ducheneaut, N. ; Moore, R. J. ; Oehlberg, L.; Thornton, J. D. ; Nickell, E. SocialTV: designing for distributed, sociable television viewing. International Journal of Human-Computer Interaction. 2008 February; 24 (2): 136154.

[7] Fine, GA (1983) Shared Fantasy: Role-playing Games as Social Worlds. Chicago: University of Chicago Press

[8] Georgen, C, Duncan, SC, and Cook, L (2015) From lurking to participatory spectatorship: Understanding affordances of the Dota 2 noob stream. CSCL 2015, Gothemburg, Sweden, 7-11 June, 2015.

[9] Hamilton, W, Garretson, O, and Kerne, A. (2014) Streaming on Twitch: Fostering participatory communities of play within live mixed media. CHI 2014, Toronto, Canada, April 26-May 1, 2014.

[10] Higginbotham, W. (1958.) Tennis For Two. Brookhaven National Laboratory.

[11] Kaytoue, M, Silva, A, Cerf, L, Meira Jr., W, Raïssi, C (2012) Watch me playing, I am a professional: a first study on videogame live streaming. $W W W 2012$ Companion, Lyon, France, 16-20 April, 2012.

[12] Miller, K (2012) Playing Along: Digital Games, YouTube, and Virtual Performance. Oxford: Oxford UP.

[13] Postigo, H (2014) The socio-technical architecture of digital labor: Converting play into YouTube money. New Media \& Society 1-18.

[14] Senft, TM (2008) Camgirls: Celebrity and Community in the Age of Social Networks. New York: Lang.

[15] Smith, D (2014) Charlie is so 'English'-like: Nationality and the branded celebrity person in the age of YouTube. Celebrity Studies 5.3: 256-74.

[16] Smith, T, Obrist, M, \& Wright, P (2013) Livestreaming changes the (video) game. 11th Proceedings of the European Conference on Interactive TV and Video, Como, Italy, 24-26 June, 2013, pp. 131-138. 